\title{
Strategies for Avoiding Neglect of Opportunity Costs by Decision-Makers
}

\author{
Jonathan Karnon ${ }^{1,2}$ (D) Andrew Partington ${ }^{1,2} \cdot$ Hossein Afzali $^{1}$ \\ Accepted: 17 August 2021 / Published online: 1 September 2021 \\ (c) The Author(s), under exclusive licence to Springer Nature Switzerland AG 2021
}

Cost-effectiveness thresholds represent decision-makers' maximum monetary valuation of a unit of outcome (typically a quality-adjusted life-year; QALY) in the context of decisions regarding the public funding of health technologies. If a technology is expected to generate additional QALYs at an incremental cost lower than the threshold, the technology is deemed to be cost effective. In this issue of Applied Health Economics and Health Policy, Lomas and colleagues note that the (policy) threshold used by the National Institute of Health and Care Excellence (NICE) is discrepant from the empirically estimated opportunity costs of the decisions made by NICE [1]. Here, we first confirm the relevance of empirical estimates of opportunity costs to informing the value of cost-effectiveness thresholds in different settings, and then build on Lomas et al.'s suggested strategy to avoid the neglect of empirical estimates of opportunity costs by decision-makers.

No decision-maker has defined a single cost-effectiveness threshold value, mainly because factors other than QALY gains influence the expected value of health technologies, including disease severity, availability of alternative treatment options, uncertainty and budget impact. Conceptually, the value of the cost-effectiveness threshold is individually considered and defined for every decision, reflecting the relevance of 'other factors' to each decision.

Empirical estimates of marginal health system productivity represent the expected QALY gains from relatively small increases in aggregate health expenditure. In England, decisions made by NICE are implemented by local health services, finding the resources required to fund a new health technology from budgets from which all other healthcare

Jonathan Karnon

jonathan.karnon@flinders.edu.au

1 Flinders Health and Medical Research Institute, Flinders University, Adelaide, SA, Australia

2 NHMRC Partnership Centre for Health System Sustainability, Sydney, Australia for defined jurisdictions are funded. This means the estimated health system marginal cost per QALY represents the expected opportunity costs of NICE decisions-decisions to fund new health technologies result in reduced health expenditure at the margin. It follows that the empirically estimated marginal cost per QALY provides a relevant reference point for defining cost-effectiveness thresholds for funding decisions that are informed by QALYs and incremental cost-effectiveness ratios (ICERs). The threshold for individual decisions can move up or down depending on the effects of 'other factors'.

In other countries, the absence of single funders of healthcare for defined jurisdictions means that the link between decisions to fund new health technologies and health expenditure at the margin is less direct. In Australia, for example, funding decisions by the Commonwealth government have little effect on funding decisions made by local health services, who are funded by State and Territory governments. Thus, whilst the available empirical estimates of the marginal effects of health expenditure are representative of the expected opportunity costs of funding decisions at a health systems level, budget silos within the health system mean the empirical estimates may not reflect actual (realworld) opportunity costs. In this context, the empirical marginal cost per QALY remains relevant because the funding of health technologies with ICERs higher than the estimated health system marginal cost per QALY is expected to reduce health system efficiency.

As Lomas et al. [1] note, NICE have stated that funding decisions should reflect the expected opportunity costs of new health technologies. The defined lower threshold value of $£ 20,000$ per QALY implies that all assessed health technologies that gain QALYs at or below that value are cost effective. Lomas et al. refer to the NICE threshold as a 'policy threshold' because the value is higher than the estimated marginal cost per QALY for the English health system ( $£ 12,936$ per QALY) [1]. Lomas et al. suggest analysts should interpret the findings of published cost-effectiveness 
analyses with respect to the relevant policy threshold as well as a threshold informed by relevant estimates of opportunity costs. Highlighting differences in implied decisions using the alternative thresholds is a strategy towards avoiding opportunity cost neglect by decision-makers.

In considering other strategies to avoid neglect of opportunity costs, one potential reason for neglect is that decisionmakers do not view available empirical estimates as sufficiently robust to inform decision-making.

Evidence to support the validity of the estimated marginal cost per QALY could include analyses of the cost effectiveness of healthcare that has been displaced as a consequence of the funding of new health technologies. Analyses showing that the weighted ICER of displaced services is close to the estimated marginal cost per QALY would be supportive of an empirical threshold. Such evidence was theorised to be observable in the UK, but studies undertaken in England and Scotland could not generate meaningful estimates of the ICERs of displaced services [2,3].

Another option is to look at evaluated health technologies for which resource allocation decisions are not made by national or central decision-makers such as NICE in England and the Pharmaceutical Benefits Advisory Committee (PBAC) in Australia, including health service delivery models for which funding decisions are made by local health services. Commonly, evaluations of such delivery models are undertaken as part of a research project for which funding for the implementation and evaluation of the health technology are provided by a research funding body, not a local health service. A commonly reported scenario in health services research is that funding is not available to support the continued provision of evaluated service delivery models [4]. Given this, evidence of the discontinuation of researchfunded health technologies with ICERs around the empirical estimate of the marginal cost per QALY would be supportive of an empirical threshold (conversely, evidence of continuation of technologies with higher ICERs would not be supportive). As Pekarsky notes [5], the best alternative use of the resources required for a new technology is not necessarily displaced current services, but alternative new uses of the required resources.

Published economic evaluations can be reviewed to identify relevant evaluated health technologies, with authors contacted to determine ongoing funding status. A barrier to the comparative assessment of published economic evaluations of health service delivery models is that outcomes are more commonly presented in natural units, or cost and QALYs are not extrapolated over an extended time horizon to estimate all important differences in costs and outcomes. For selected evaluations-for example, those with the most robust clinical evidence-modelling could be undertaken to extrapolate reported differences in costs and outcomes. As an example, a published economic evaluation of a physiotherapy-led intervention for frail older people reported an incremental cost per person transitioning from frailty of $\mathrm{A} \$ 15,955$ over a 1-year time horizon, and an implied incremental cost per QALY gained of over A $\$ 200,000$ [6]. Using a cost-effectiveness model, the 1-year findings were extrapolated to a lifetime horizon, for which the mean estimated incremental cost per QALY gained was A $\$ 8129$ [7].

A thorough assessment of the clinical and cost effectiveness of relevant evaluated health technologies would follow a similar process to that undertaken by bodies such as NICE and PBAC. As noted, conceptually, cost-effectiveness thresholds are defined for each funding decision, adjusting a reference threshold value for the effects of other factors. The aim would be to specify a mean ICER for relevant evaluated health technologies, with accompanying assessments of the uncertainty around the mean ICER and of other factors that could influence the cost-effectiveness threshold. The findings could be qualitatively compared with assessments of health technologies for which funding decisions have been made by bodies such as NICE and PBAC to inform the validity of reference cost-effectiveness thresholds based on empirical estimates of the marginal cost per QALY, and judgements on whether and to what extent stated or implied policy thresholds are too high (or too low).

Continuing improvements in data and methods to estimate the marginal cost per QALY - for example, more routine collection of Patient Reported Outcomes-should increase acceptance and use of such estimates in decisionmaking. Comparative evidence on the cost effectiveness and funding of health technologies for which funding decisions are made in different decision-making contexts may further support the validity and uptake of empirical estimates of the marginal cost per QALY as a reference ICER for decision-makers.

\section{Declarations}

Funding No sources of funding were used to prepare this article.

Conflict of interest Jonathan Karnon is a member of the economic sub-committee of the Pharmaceutical Benefits Advisory Committee in Australia. Hossein Afzali is a member of the evaluation sub-committee of the Medical Services Advisory Committee in Australia. Andrew Partington has no known conflicts of interest.

\section{References}

1. Lomas J, Ochalek J, Faria R. Avoiding opportunity cost neglect in cost-effectiveness analysis for health technology assessment. Appl Health Econ Health Policy. 2021

2. Appleby J, Devlin N, Parkin D, Buxton M, Chalkidou K. Searching for cost effectiveness thresholds in the NHS. Health Policy. 2009;91(3):239-45. 
3. Sussex J, Hughes D, Devlin N. Opportunity costs and local health service spending decisions: a qualitative study from Wales. BMC Health Serv Res. 2016;25(16):103.

4. Fradgley EA, Karnon J, Roach D, Harding K, Wilkinson-Meyers L, Chojenta C, et al. Taking the pulse of the health services research community: a cross-sectional survey of research impact, barriers and support. Aust Health Rev. 2019;44(1):160-7.

5. Pekarsky BA. The "New Drug Reimbursement" game. Cham: Adis; 2015. p. 127-62.

6. Fairhall N, Sherrington C, Kurrle SE, Lord SR, Lockwood K, Howard K, Hayes A, Monaghan N, Langron C, Aggar C, Cameron
ID. Economic evaluation of a multifactorial, interdisciplinary intervention versus usual care to reduce frailty in frail older people. J Am Med Dir Assoc. 2015;16(1):41-8.

7. Karnon J, Afzali HH, Putro GV, Thant PW, Dompok A, Cox I, Chikhwaza OH, Wang X, Mwangangi MM, Farransahat M, Cameron I. A cost-effectiveness model for frail older persons: development and application to a physiotherapy-based intervention. Appl Health Econ Health Policy. 2017;15(5):635-45. 